\title{
Desarrollo de un modelo matemático de diferencias finitas para el análisis del campo de temperaturas en la soldadura por arco de chapas finas de acero inoxidable $e^{(*)}$
}

\author{
E. J. Martínez-Conesa*, M. Estrems* y V. Miguel**
}

\begin{abstract}
Resumen
En este trabajo se desarrolla un método de diferencias finitas para calcular el campo de temperaturas en la zona afectada por el calor en la soldadura de dos chapas de acero inoxidable AISI 304, soldadas mediante el procedimiento GTAW. Se ha desarrollado un programa informático implementado en libros de cálculo MS-Excel con Visual Basic para Aplicaciones (VBA). Los experimentos modelizados a través de la aplicación numérica predicen el comportamiento térmico de un procedimiento de soldadura. Para la validación del modelo matemático se ha desarrollado un procedimiento experimental para medir el campo de temperaturas en la zona cercana al cordón de soldadura de un acero inoxidable de espesor inferior a $3 \mathrm{~mm}$. Este procedimiento experimental se puede utilizar para otros tipos de metales y aleaciones y para otros procedimientos de soldadura por arco eléctrico como el MIG/MAG, SMAW y FCAW. Los datos obtenidos con estos ensayos se han utilizado para validar el cálculo del campo de temperaturas mediante la aplicación numérica de diferencias finitas. Con estos experimentos se ha realizado el ajuste del modelo matemático, y las diferencias que existen entre las curvas experimentales y teóricas son debidas a las pérdidas de calor por convección y radiación, que no se han estimado en el modelo de simulación. Con este sencillo modelo el proyectista podrá calcular los ciclos térmicos del proceso y predecir el campo de temperaturas en las proximidades del cordón.
\end{abstract} peraturas; Zona afectada por el calor.

\section{A mathematical approach based on finite differences method for analyzing the temperature field in arc welding of stainless steel thin sheets}

\begin{abstract}
This work develops a finite difference method to evaluate the temperature field in the heat affected zone in butt welding applied to AISI 304 stainless steel thin sheet by GTAW process. A computer program has been developed and implemented by Visual Basic for Applications (VBA) in MS-Excel spreadsheet. The results that are obtained using the numerical application foresee the thermal behaviour of arc welding processes. An experimental methodology has been developed to validate the mathematical model that allows to measure the temperature in several points close to the weld bead. The methodology is applied to a stainless steel sheet with a thickness lower than $3 \mathrm{~mm}$, although may be used for other steels and welding processes as MIG/MAG and SMAW. The data which has been obtained from the experimental procedure have been used to validate the results that have been calculated by the finite differences numerical method. The mathematical model adjustment has been carried out taking into account the experimental results. The differences found between the experimental and theoretical approaches are due to the convection and radiation heat losses, which have not been considered in the simulation model. With this simple model, the designer will be able to calculate the thermal cycles that take place in the process as well as to predict the temperature field in the proximity of the weld bead.
\end{abstract}

Keywords

Numerical simulation of temperature field; Arc welding; Finite differences method; Temperature measure; Heat affected zone.

\footnotetext{
(•) Trabajo recibido el día 13 de mayo de 2010 y aceptado en su forma final el día 8 de julio de 2010.

* Departamento Arquitectura y Tecnología de la Edificación, Escuela de Arquitectura e Ingeniería de la Edificación, C/ Alfonso XIII, 52 , 30203 Cartagena, España.

** Departamento de Mecánica Aplicada e Ing. de Proyectos, Escuela de Ingenieros Industriales de UCLM, Avda. de España, s/n 02005, Albacete, España.
} 


\section{INTRODUCCIÓN}

Toda estructura metálica se forma a partir de diferentes elementos interdependientes que se unen entre sí, siendo el objetivo de esta unión el de asegurar la continuidad entre las diferentes piezas. La unión se puede realizar mediante diferentes métodos entre los que podemos mencionar: uniones remachadas, uniones con tornillos, uniones con adhesivos y uniones soldadas. De entre todas ellas, la soldadura destaca desde hace tiempo. La razón de ello, es que la soldadura presenta ventajas muy favorables frente a los otros tipos de unión como son: disminución del peso, facilidad de diseño, facilidad y rapidez de montaje y facilidad de reparación. Sin embargo, el uso de uniones soldadas presenta también algunos inconvenientes debidos a la falta de conocimientos por parte del diseñador y a la falta de preparación de los que en última instancia ejecutan la unión soldada. Dichos inconvenientes, entre otros son: el control de ejecución de la obra debe ser más estricto, los operarios deben de ser cualificados, las uniones no son desmontables y se produce una generación de tensiones debido al ciclo térmico de soldeo que merma la capacidad resistente de la pieza o de la estructura en general. En las uniones soldadas, como consecuencia directa del aporte de calor no uniforme y el posterior enfriamiento, se desarrolla un complejo estado de tensiones térmicas residuales que puede disminuir directa o indirectamente la capacidad portante de la estructura. Por lo tanto, la predicción y control de la distribución de temperaturas en la zona de la junta soldada es crucial en la edificación.

Debido a la complejidad de los procesos físicos involucrados en el procedimiento de soldadura no ha podido encontrarse una solución matemática que describa y resuelva este problema. Además, es muy complicado obtener experimentalmente los datos del campo de temperaturas en todos los puntos de la zona afectada térmicamente en un conjunto soldado. Por lo tanto, las simulaciones numéricas juegan un papel fundamental en el análisis de este tipo de estructuras. En estas simulaciones numéricas, el modelado del material es uno de los problemas clave. En la práctica son difíciles de obtener datos completos de las propiedades del material en función de la temperatura, más aún para las altas temperaturas alcanzadas en el procedimiento de soldadura, en el que hay zonas donde se alcanza una temperatura bastante superior a la de fusión del material. En este trabajo de investigación, para resolver este problema se ha desarrollo un modelo de simulación del campo de temperaturas en la zona cercana al cordón de soldadura en un acero inoxidable estructural. Los resul- tados obtenidos son las temperaturas y los flujos de calor generados en la junta soldada.

Por tanto, el objetivo principal de esta investigación es desarrollar un método de diferencias finitas para el cálculo del ciclo térmico de soldeo, en función de las propiedades térmicas del material, los parámetros geométricos del metal base y los parámetros de soldeo: intensidad, tensión, velocidad de soldeo y rendimiento del procedimiento de soldadura. Este método se ha implementado en VBA y ha sido validado experimentalmente con oportunos experimentos de soldadura, y como aplicación del método, se puede calcular la dimensión de la ZAT y el cálculo del tiempo de enfriamiento del cordón de soldadura de un acero inoxidable.

\subsection{Antecedentes de la simulación numérica del ciclo térmico de soldeo}

Con este trabajo se destaca la importancia de utilizar modelos de simulación basados en métodos numéricos, para que el ingeniero pueda predecir el campo de temperaturas en la junta de unión de un acero inoxidable estructural.

En los procedimientos de soldadura por arco eléctrico, el aporte de energía se realiza en una zona localizada alcanzándose temperaturas muy superiores a la temperatura de fusión del material base. Las altas diferencias de temperatura que se establecen entre la zona de aplicación del calor y las zonas adyacentes a ésta, junto con la buena conductividad térmica que presentan los metales a soldar originará un importante flujo térmico que condicionará las estructuras y propiedades resultantes tanto en el cordón de soldadura como en las zonas cercanas a él. Cuando se analizan los efectos perjudiciales que el ciclo térmico de soldeo puede producir sobre la pieza soldada, el interés no se centra necesariamente en la zona de fusión o cordón de soldadura, sino también en la zona cercana a éste, denominada zona afectada térmicamente (ZAT), que suele presentar un grado de debilidad mayor en sus propiedades mecánicas que el propio cordón. Por lo tanto, el conocimiento de la evolución de la temperatura en los alrededores del cordón de soldadura, durante el proceso de soldeo y hasta que la temperatura sea inferior a aquélla que provoca cambios en las propiedades del material, es vital para determinar el grado de debilidad en el que ha quedado la ZAT, y para establecer estrategias que minimicen estos efectos térmicos mediante precalentamientos del material base, tratamientos térmicos, etc. Para conseguir este conocimiento del campo de temperaturas y evitar la necesidad de recurrir a ensayos experimentales, siempre 
costosos, se puede hacer uso de métodos analíticos y numéricos que solucionen las ecuaciones diferenciales de la conducción del calor.

Los métodos analíticos ${ }^{[1 \text { y } 2]}$ son los más atractivos puesto que de casos simples es posible obtener soluciones y conclusiones de las que el ingeniero puede servirse para ajustar los parámetros de soldadura, y evitar los defectos indeseados que aparecen en la ZAT. Una de las primeras soluciones que se pueden

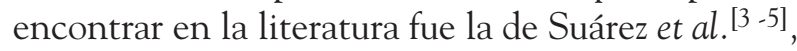
que proponen soluciones particulares de la ecuación de conducción del calor para flujo bidimensional y que aplicaron con éxito a la soldadura, determinando el campo de temperaturas en el entorno del cordón. Nguyen et al..$^{[6 \text { y } 7]}$ presentaron dos trabajos en los que partiendo de una fuente de calor de doble elipsoide, determinaron la forma del baño líquido y su evolución a lo largo del proceso de soldeo. Ambos métodos tienen el inconveniente de no tener en cuenta el calor latente de cambio de estado, lo cual afecta claramente a la difusión del calor.

Desde la aparición de programas de elementos finitos (ANSYS, ALGOR, NASTRAN, etc.), una solución muy recurrida ha sido la utilización de estos programas para el análisis de la evolución del ciclo térmico en el proceso de soldadura, tal como se puede ver en los trabajos realizados por Goldak et al. ${ }^{[8-10]}$. Sin embargo, las dificultades de mallado y la complejidad de los fenómenos que aparecen durante el proceso de soldadura ha hecho que se hayan desarrollado métodos numéricos que tienen en cuenta factores tales como la existencia de campos electromagnéticos, la transferencia de metal del electrodo al cordón y la interfase líquido-sólido en éste. Estos métodos se centran en la física específica del proceso. Así Xu et al. ${ }^{[11]}$ han modelizado el proceso de transferencia de metal en cortocircuito en soldadura por arco mediante el método de volúmenes finitos, y Debroy et al. ${ }^{[12]}$ han obtenido la modelización del proceso de electroescoria mediante el método de diferencias finitas tridimensional.

Sin entrar en la complejidad de los métodos anteriormente descritos, y frente a soluciones analíticas ideales como las aportadas por Suárez et al..$^{[4]}$ y Nguyen et al. ${ }^{[6]}$, el método de diferencias finitas presentado tiene en cuenta los cambios de estado determinando la fracción líquida en cada punto del cordón, además de una mayor flexibilidad en la definición de la fuente de calor. Algunas ventajas del procedimiento implementado, en comparación con otros métodos numéricos tales como el de los elementos finitos, son que proporciona unos tiempos de resolución mucho menores, su uso e implementación es muy sencillo y puede ser utilizado en cualquier ordenador que tenga instalado MS-Excel.

La originalidad de este método numérico se basa en la modificación realizada en la resolución por
Crank-Nicholson de la ecuación de conducción del calor, ya que se ha tenido en cuenta el calor producido en el cambio de estado de sólido a líquido y de líquido a sólido que se produce en la soldadura de un acero inoxidable. Una recopilación de los trabajos más recientes en donde se describen técnicas modernas de cálculo numérico para analizar estos problemas aparecen citadas en las referencias ${ }^{[13 \text { y } 14]}$.

\section{ANÁLISIS MATEMÁTICO DEL FLUJO DE CALOR EN PROCESOS DE SOLDADURA}

El análisis matemático del flujo de calor en procesos de soldadura consiste en solucionar la ecuación diferencial de conducción del calor. Para solucionarla se asume que no hay pérdidas al medio circundante y que el calor generado por el proceso de deformación es ignorado.

La ecuación fundamental de conducción del calor desarrollada es la indicada en la ecuación (1).

$$
\frac{\partial \mathrm{T}}{\partial t}=\frac{k}{\rho c_{p}}\left(\frac{\partial^{2} \mathrm{~T}}{\partial \mathrm{x}^{2}}+\frac{\partial^{2} \mathrm{~T}}{\partial \mathrm{y}^{2}}+\frac{\partial^{2} \mathrm{~T}}{\partial \mathrm{z}^{2}}\right)+\frac{\partial \alpha}{\partial T}\left[\left(\frac{\partial \mathrm{T}}{\partial \mathrm{x}}\right)^{2}+\left(\frac{\partial \mathrm{T}}{\partial \mathrm{y}}\right)^{2}+\left(\frac{\partial \mathrm{T}}{\partial z}\right)^{2}\right]
$$

Siendo $T$ la temperatura en un determinado punto de coordenadas $X, Y, Z$ del sólido; $\rho, \mathrm{C}_{p}, k$ y $\alpha$ corresponden a la densidad, el calor especifico, la conductividad térmica y la difusividad térmica del material respectivamente.

La ecuación (1) se aplica sobre la geometría de la soldadura y con unas ciertas condiciones de contorno como son la temperatura inicial fijada o flujo de calor en los bordes. Solucionándola, se obtendría la distribución de temperaturas que se genera durante el procedimiento de soldeo.

Para el acero AISI 304 se ha considerado que sus propiedades térmicas varían con el aumento de la temperatura ${ }^{[15]}$ y se han ajustado funciones matemáticas sobre cada una de las propiedades del material con el fin de procesar numéricamente la información que proporcionan.

En las ecuaciones (2), (3) y (4) se indica la variación de la conductividad térmica, calor específico y densidad del AISI 304 con la temperatura.

$$
\begin{gathered}
K(T)=-4 E^{-0.6} T^{2}+0.0155 T+15.081 \\
C_{P}(T)=-3 E^{-0.5} T^{2}+0.2104 T+486.6 \\
\rho(T)=-8 E^{-0.8} T^{2}-0.0004 T+7.88
\end{gathered}
$$


Esta variación de las propiedades físicas del material con la temperatura lleva a la necesidad de resolver un problema no-lineal, requiriéndose en este caso un proceso iterativo de análisis. Una forma de solucionar este problema es aplicando el método de Crank-Nicholson a la ecuación diferencial de conducción del calor ${ }^{[16]}$.

Si se particulariza para flujo de calor bidimensional, la ecuación de conducción de calor se simplifica conforme a lo indicado en la ecuación (5).

$$
\frac{\partial \mathbf{T}}{\partial \mathrm{t}}=\frac{\mathbf{k}}{\rho \mathbf{c}_{p}}\left(\frac{\partial^{2} \mathbf{T}}{\partial \mathrm{x}^{2}}+\frac{\partial^{2} \mathrm{~T}}{\partial \mathbf{y}^{2}}\right)+\frac{\partial \alpha}{\partial T}\left[\left(\frac{\partial \mathrm{T}}{\partial \mathrm{x}}\right)^{2}+\left(\frac{\partial \mathrm{T}}{\partial \mathrm{y}}\right)^{2}\right]
$$

Simplificando y operando la ecuación (5), se obtiene la ecuación (6). El factor $\mathrm{F}_{0}$ se define en la ecuación (7).

$$
\begin{gathered}
\mathrm{T}_{\mathrm{n}}^{\mathrm{p}+1}=\left(\frac{1-2 \mathrm{~F}_{0}}{1+2 \mathrm{~F}_{0}}\right) \mathrm{T}_{\mathrm{n}}^{\mathrm{p}}+ \\
\frac{\mathrm{F}_{0}}{\left(1+2 \mathrm{~F}_{0}\right)\left(\mathrm{T}_{\mathrm{B}}^{\mathrm{p}+1}+\mathrm{T}_{\mathrm{W}}^{\mathrm{p}+1}+\mathrm{T}_{\mathrm{B}}^{\mathrm{p}}+\mathrm{T}_{\mathrm{W}}^{\mathrm{p}}+\mathrm{T}_{\mathrm{N}}^{\mathrm{p}+1}+\mathrm{T}_{\mathrm{S}}^{\mathrm{p}+1}+\mathrm{T}_{\mathrm{N}}^{\mathrm{p}}+\mathrm{T}_{\mathrm{S}}^{\mathrm{p}}\right)} \\
F_{o}=\frac{k \Delta t}{\rho C_{p} \Delta X^{2}}
\end{gathered}
$$

\subsection{Calor del cambio de estado sólido- líquido}

La principal aportación del presente trabajo de investigación es la singularidad que aparece en el cambio de estado, que será móvil y llevará asociada una deposición y absorción de la energía. Debido a la gran inercia térmica, los sistemas con cambio de estado son muy utilizados para el almacenamiento térmico. En los modelos térmicos de soldadura, sólo se utiliza el cambio de estado de sólido a líquido y de líquido a sólido para evitar grandes variaciones de volumen cuando se produce la solidificación del cordón de soldadura. No obstante, se ha de prever un volumen de compensación no sólo para las dilataciones sino para el cambio de volumen asociado al cambio de estado.

En este trabajo, se analiza la transmisión de calor en un acero inoxidable austenítico, considerado como una sustancia pura caloríficamente perfecta, esto es, que el calor específico a presión constante, Cp, no es función de la temperatura, siendo un valor constante. El material se caracteriza por las capacidades térmicas $\mathrm{c}_{\mathrm{s}}$ y $\mathrm{c}_{1}$ y las conductividades térmicas $\mathrm{k}_{\mathrm{s}}$ y $\mathrm{k}_{\mathrm{l}}$ del sólido y del líquido respectivamente. Para simplificar la formulación y no introducir efectos de convección se asume que $\rho_{\mathrm{s}}=\rho_{\mathrm{l}}$.
El balance de calor, $Q$, aportado y liberado por el cambio de estado líquido-sólido para una fracción $x$ de material licuado en el intervalo correspondiente al tiempo de discretización se expresa en la ecuación (8), en la que $V$ representa la tensión de soldeo, I la intensidad de corriente, $\rho$ la densidad del material base, $\Delta H$ la variación de entalpía en el cambio de estado y $\beta$ el rendimiento térmico del proceso de soldadura.

$$
Q=\beta V I-\rho \Delta H_{x}
$$

De este modo, la ecuación diferencial de la conducción de calor en flujo bidimensional, teniendo en cuenta el calor producido en el cambio de estado, Q, se establece conforme a la ecuación (9).

$$
\frac{\partial \mathrm{T}}{\partial \mathrm{t}}=\frac{\mathbf{k}}{\rho C_{p}}\left(\frac{\partial^{2} \mathrm{~T}}{\partial \mathrm{x}^{2}}+\frac{\partial^{2} \mathrm{~T}}{\partial \mathrm{y}^{2}}\right)+\frac{\partial \alpha}{\partial T}\left[\left(\frac{\partial \mathrm{T}}{\partial \mathrm{x}}\right)^{2}+\left(\frac{\partial \mathrm{T}}{\partial \mathrm{y}}\right)^{2}\right]+\frac{Q}{\rho C_{p}}
$$

Si aplicamos la discretización de CrankNicholson, teniendo en cuenta el calor de cambio de estado se obtiene la ecuación (10).

$$
\begin{gathered}
\mathrm{T}_{\mathrm{n}}^{p+1}=\left(\frac{1-2 \mathrm{~F}_{0}}{1+2 \mathrm{~F}_{0}}\right) \mathrm{T}_{\mathrm{n}}^{\mathrm{p}}+ \\
\frac{\mathrm{F}_{0}}{\left(1+2 \mathrm{~F}_{0}\right)\left(\mathrm{T}_{\mathrm{B}}^{p+1}+\mathrm{T}_{W}^{p+1}+\mathrm{T}_{\mathrm{B}}^{\mathrm{p}}+\mathrm{T}_{W}^{p}+\mathrm{T}_{\mathrm{N}}^{p+1}+\mathrm{T}_{S}^{p+1}+\mathrm{T}_{\mathrm{N}}^{\mathrm{p}}+\mathrm{T}_{\mathrm{S}}^{p}\right)}+\frac{Q}{1+2 \mathrm{~F}_{0}}
\end{gathered}
$$

\subsection{Condiciones iniciales y de contorno}

Las condiciones iniciales del proceso se materializan en la temperatura inicial de la chapa, previa al proceso de soldadura. Según esto, la temperatura inicial se considera la temperatura ambiente en procesos de soldadura sin precalentamiento. En el caso en que se suelde con precalentamiento, se considera ésta como temperatura inicial.

Se usa la condición frontera de Dirichlet donde se especifica en la frontera un valor fijo de la temperatura, en este caso se ha aplicado para la frontera norte. La otra condición de contorno que se aplica es la condición frontera de Neuman, que específica en la frontera el valor de la derivada de la temperatura, en este caso se aplica a la frontera este, oeste y sur, en donde se consideran condiciones adiabáticas y, por lo tanto, gradientes nulos de temperaturas.

\section{PROCEDIMIENTO DE CÁLCULO}

La implementación del procedimiento de cálculo descrito se ha realizado utilizando hojas de cálculo MS-Excel con Visual Basic para aplicaciones (V.B.A), 
dada la sencillez y gran difusión de estos programas. Este tipo de formato facilita la intercambiabilidad con otros programas y su interrelación con otros libros de cálculo utilizados, así como la visualización gráfica de los resultados. En la tabla I se establece la secuenciación de libros de cálculo considerados en los que se distribuyen las tareas que son realizadas para la cumplimentación del procedimiento de análisis numérico.

Para introducir los datos se utiliza una primera hoja de cálculo, que una vez implementada, es la que contiene las propiedades térmicas y físicas del material, geometría, tensión, intensidad y velocidad de soldeo, tamaño de discretización, y patrón de aporte de calor. El patrón de aporte de calor corresponde al perfil de la fuente de calor ya discretizado, para esto se ha utilizado la modelización de aporte de calor realizada por Goldak ${ }^{[8]}$.

A partir de los datos de entrada, en diferentes hojas de cálculo queda representada la discretización de la chapa a soldar. Cada celda de la correspondiente hoja, representa un punto de discretización de dicho material. Cada una de las hojas de cálculo representan el valor de una característica, sea temperatura o fracción líquida en el instante $n$ y $n+1$, y la posición de la fuente de calor en el instante $n+1$. El cálculo de las temperaturas y fracciones líquidas se

\section{Tabla I. Esquema de la distribución de hojas de cálculo}

\section{Table I. Scheme of the Excel sheets distribution}

\section{Programa de simulación ciclo térmico soldeo}

Datos geométricos, parámetros de soldeo y propiedades material

Temperatura en el instante "i"

Temperatura en el instante " $\mathrm{i}+1$ "

Fracción líquida en el instante "i”

Fracción líquida en el instante “i+1”

Cálculo de aportación de calor Goldak

Resultados: Distribución de temperaturas

Cálculo ZAT

Cálculo $\mathrm{t}_{8 / 5}$ realiza en el instante $n+1$. En cuanto la solución converge conforme al error definido en el diseño del programa, se copian los resultados en el instante $n+1$ en las hojas del instante $n$, se registran los datos en la hoja "Resultados", se reposiciona el aporte de calor, y se procede al cálculo del siguiente intervalo de tiempo.

En la hoja "Resultados" queda reflejada la evolución de temperatura de los puntos de interés y su representación gráfica.

El mismo libro de Excel tiene un proyecto VBA que contiene un módulo de programación, el cual, usando los objetos de las hojas anteriormente señaladas, aplica fórmulas y realiza los procedimientos para calcular todo el proceso.

\section{PROCEDIMIENTO EXPERIMENTAL}

El objetivo fundamental del procedimiento experimental es realizar una validación del método de diferencias finitas y de esta forma presentar un modelo matemático que le permita al ingeniero calcular unas predicciones futuras del campo de temperaturas antes de realizar la unión soldada.

El procedimiento de soldeo empleado es el "Gas Tungsten Arc Welding" (GTAW), soldadura por arco eléctrico con protección de gas inerte con un electrodo no consumible. El caudal de gas utilizado es $12 \mathrm{l} / \mathrm{min}$, y la soldadura se ha realizado sin material de aportación. La posición de soldeo es horizontal y sin preparación de bordes. El electrodo empleado es de tungsteno aleado con lantano de diámetro 1,6 $\mathrm{mm}$. Se ha empleado corriente continua con polaridad directa. Los datos del proceso de soldeo son coincidentes con los considerados en las simulaciones anteriores.

La temperatura de la chapa se ha medido mediante el empleo de termopares y el registro de temperaturas se ha efectuado mediante un sistema de adquisición de datos por ordenador. Los termopares utilizados para medir las temperaturas del metal base son los de tipo $\mathrm{K}$, formados por un par trenzado de hilo sólido de $0,2 \mathrm{~mm}$ de diámetro y con una precisión de $0,5^{\circ} \mathrm{C}$ en el rango de funcionamiento de $-75^{\circ} \mathrm{C}$ a $1.200^{\circ} \mathrm{C}$. Se ha procurado establecer un buen contacto entre el extremo del termopar y el material base. Para ello, con un taladro se han realizado unos orificios de $1 \mathrm{~mm}$ de diámetro para la introducción del extremo del termopar y de esta manera asegurar el contacto con el metal base, tal y como se aprecia en la figura 1 .

El registro de datos se ha efectuado con un registrador comercial TC-08, conectado a un PC que permite adquirir, y visualizar la evolución de los datos de 8 termopares. Para registrar todos los datos de temperaturas que se registran, se utiliza un software comercial Picolog for Windows 5.15.6. que tiene 


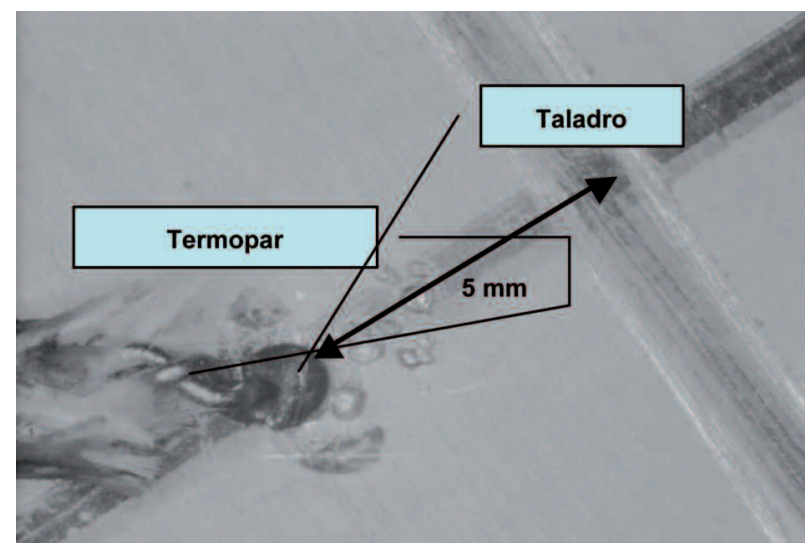

Figura 1. Posicionamiento de un termopar.

Figure 1. Thermocouple position.

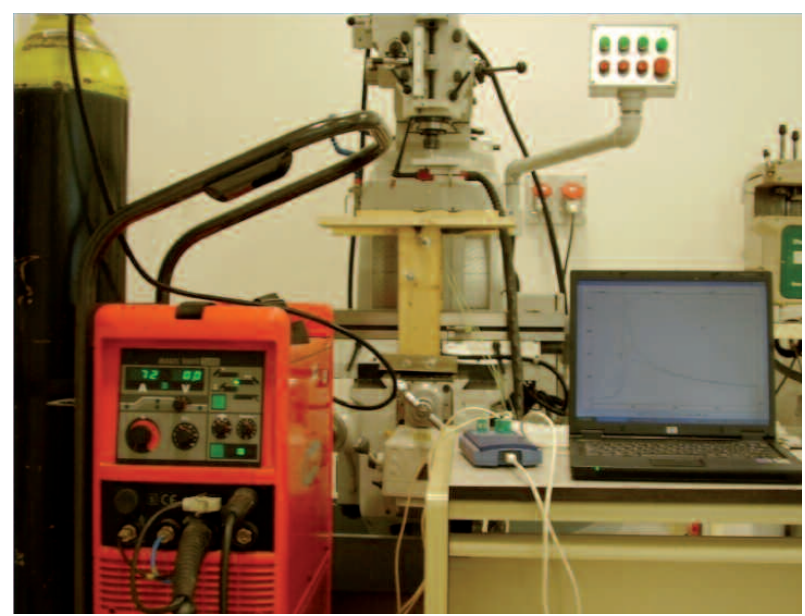

Figura 2. Equipamiento utilizado en procedimiento experimental.

Figure 2. Experimental procedure.

capacidad de 20 bits, con un tiempo de muestreo de $500 \mathrm{~s}$ y frecuencia de muestro de $1 \mathrm{~s}$.

En la figura 2 se indica el montaje de los elementos que intervienen en el procedimiento experimental. El metal base se fija a unos soportes de madera para disminuir las posibles deformaciones que se producen durante el proceso de soldeo. A continuación se posiciona en la bancada de una fresadora y se coloca el portaeléctrodo de la máquina de soldadura en el cabezal de la fresadora, se programan ambas máquinas, la fresadora y la máquina TIG, de esta forma la velocidad y los demás parámetros de soldeo están controlados durante los experimentos. Durante el procedimiento de soldeo y posterior enfriamiento se almacenan las temperaturas registradas por los termopares.

En la figura 3 se indica el método de ensayo utilizado en este procedimiento experimental.

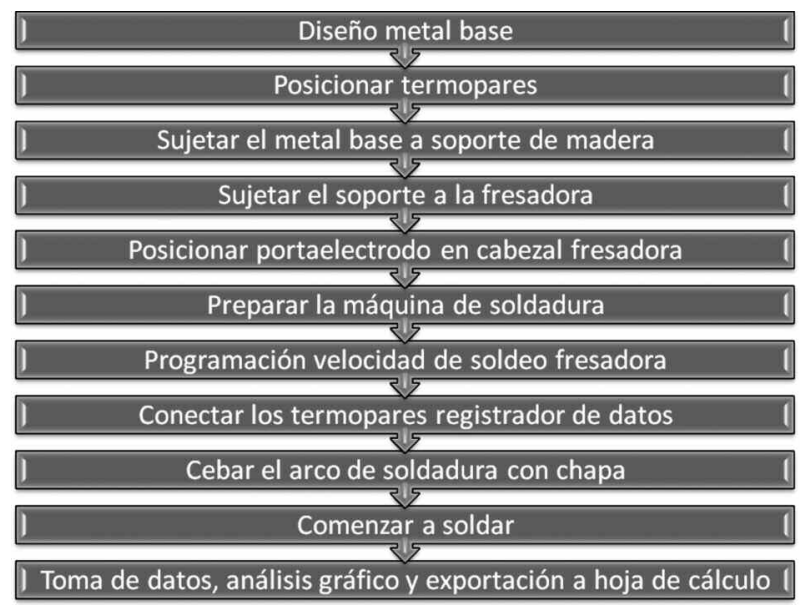

Figura 3. Método de Ensayo.

Figure 3. Experimental method.

\section{APLICACIÓN DEL MÉTODO A LA SOLDADURA A TOPE DE CHAPA FINA DE ACERO INOXIDABLE AISI 304, MEDIANTE EL PROCESO TIG. DISCUSIÓN DE RESULTADOS}

Como ejemplo de aplicación del método se presentan los datos para la simulación de un caso particular, consistente en la soldadura de chapa de $3 \mathrm{~mm}$ de espesor empleando una velocidad de soldeo de $0,0033 \mathrm{~m} / \mathrm{s}$, así como valores de tensión e intensidad de $20 \mathrm{~V}$ y $120 \mathrm{~A}$, respectivamente. El procedimiento de soldeo empleado es GTAW, la soldadura se realiza en posición horizontal y la posición de los bordes es a tope. Los datos de partida corresponden con valores experimentales de intensidad, tensión, velocidad y rendimiento térmico de soldeo para la unión de chapa de acero inoxidable AISI 304. El cordón de soldadura se realiza a lo largo de toda su longitud y como el problema es simétrico, en la hoja de cálculo se representa sólo una de las chapas a soldar.

La discretización bidimensional tiene una relación de aspecto de uno es a uno. El intervalo de tiempo se ha tomado lo suficientemente pequeño para disminuir el error parabólico y con un valor que depende de la velocidad y de la discretización.

Si se representa en tres dimensiones los datos obtenidos en la hoja de resultados, correspondiente al caso de aplicación indicado, se obtiene la grafica de la figura 4. En dicha figura, el eje $\mathrm{Z}$ corresponde a la temperatura alcanzada en ${ }^{\circ} \mathrm{C}$; en el eje $\mathrm{X}$ se representa la distancia en $\mathrm{mm}$ de las diferentes isotermas al centro del cordón de soldadura; por último, el eje Y representa el tiempo transcurrido en segundos. 


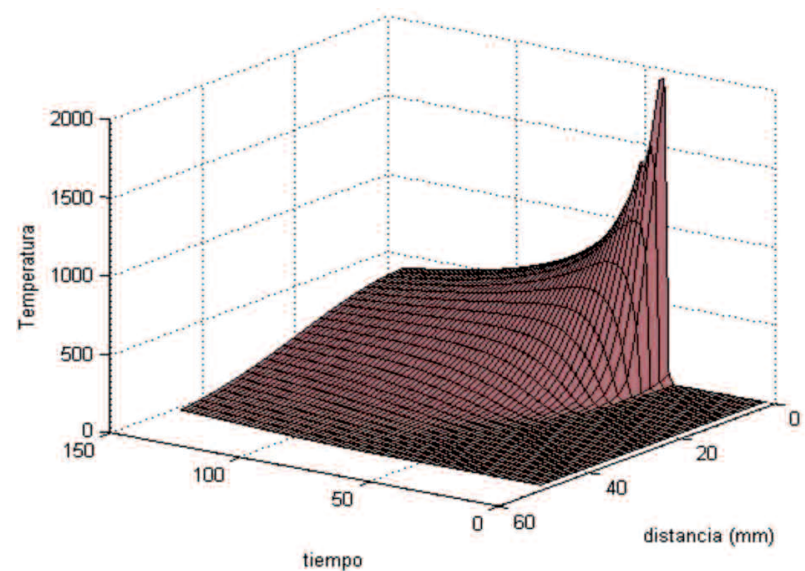

Figura 4. Simulación del ciclo térmico de soldeo tridimensional.

\section{Figure 4. Three-dimensional simulation of the welding heat cycle.}

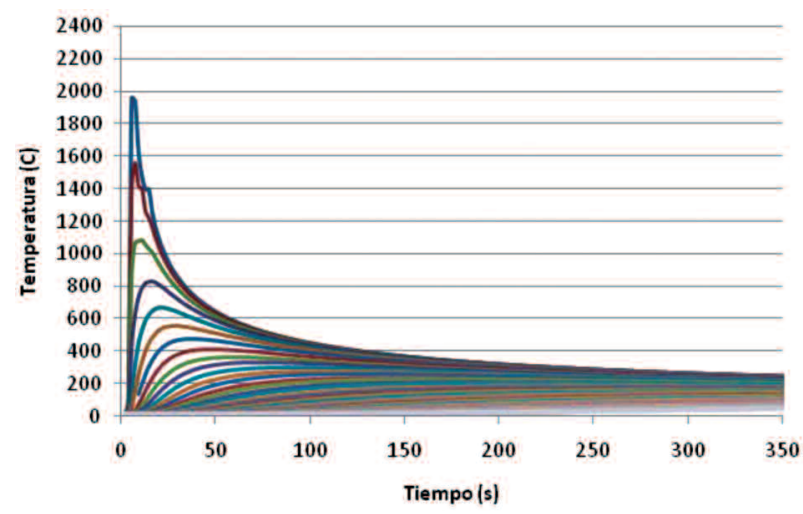

Figura 5. Simulación del ciclo térmico de soldeo (2D).

\section{Figure 5. Welding heat cycle simulation (twodimensional).}

Si se representan los resultados en dos dimensiones se obtiene la evolución temporal de las temperaturas en la zona central de la chapa, tomada en puntos de la línea perpendicular al eje del cordón de soldadura, que es la indicada en la figura 5. Cada curva de la figura 5 indica el ciclo térmico de soldeo a diferentes distancias del eje de fusión de la junta soldada. El ejemplo correspondiente a dicha figura, corresponde a una discretización del mallado de la chapa de $2 \mathrm{~mm}$. Así, la distancia de cada isoterma al eje del cordón será de 2, 4, 6, 8 y 10 mm, etc. Como se observa en la figura 5 , se distinguen tres etapas térmicas diferentes. La primera corresponde al aumento de la temperatura que se produce en los primeros instantes de la operación de soldeo. La segunda fase

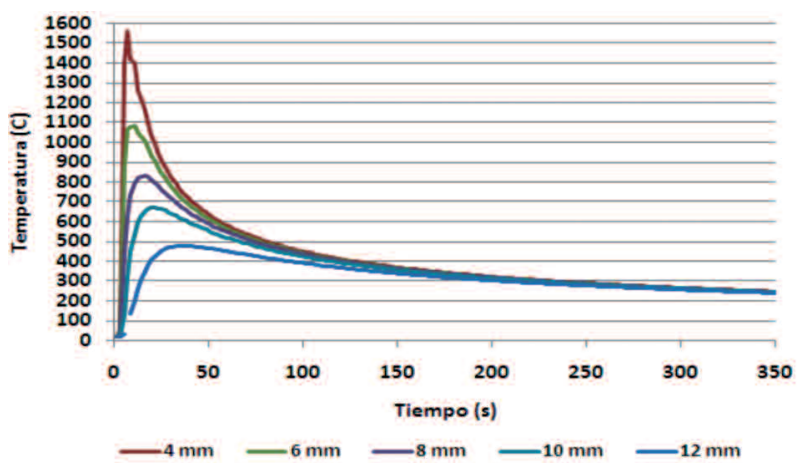

Figura 6. Simulacion de ZAT.

Figure 6. Simulation of the HAZ.

corresponde a una distribución de temperaturas estacionaria en un sistema de coordenadas que se mueva con la fuente de calor. Finalmente, existe una fase térmica en la que la temperatura permanece uniforme a partir del instante en que el arco eléctrico desaparece.

Partiendo de datos experimentales se puede afirmar que solo una franja de la chapa alcanza los $500{ }^{\circ} \mathrm{C}$, considerada esta temperatura como crítica desde el punto de vista de la precipitación de carburos en este acero inoxidable. Según esto, el interés del análisis se centra en las curvas de temperatura que se encuentran por encima de dicho valor, tal y como se detalla en la figura 6 . De este modo, se calcula la dimensión de la ZAT, la velocidad de enfriamiento de cada punto, la cantidad de metal base fundido, y se estima la severidad del cambio en la microestructura y otras propiedades del cordón de soldadura. Todo esto afecta al comportamiento de la junta soldada.

Como se observa en la figura 6, la zona del campo de temperaturas que está por encima de $1.400^{\circ} \mathrm{C}$, es la parte que pertenece al cordón de soldadura. $\mathrm{La}$ zona por debajo de $500{ }^{\circ} \mathrm{C}$ corresponde a puntos del metal base no afectado térmicamente. Entre ambas temperaturas se establece la ZAT.

La figura 7 representa gráficamente las temperaturas máximas de cada uno de los puntos del metal base en función de la distancia al eje del cordón de soldadura. En ella se indica que el inicio de la ZAT se produce a la distancia de $4,5 \mathrm{~mm}$ y el final está a $14,5 \mathrm{~mm}$ del eje del cordón de soldadura. En este caso se puede concluir, que la dimensión de la ZAT es de $10 \mathrm{~mm}$. Como cabía esperar, también se observa que la temperatura máxima disminuye con el aumento de la distancia al cordón, que el tiempo necesario para alcanzar la temperatura máxima en cada punto aumenta con dicha distancia así como la velocidad de 


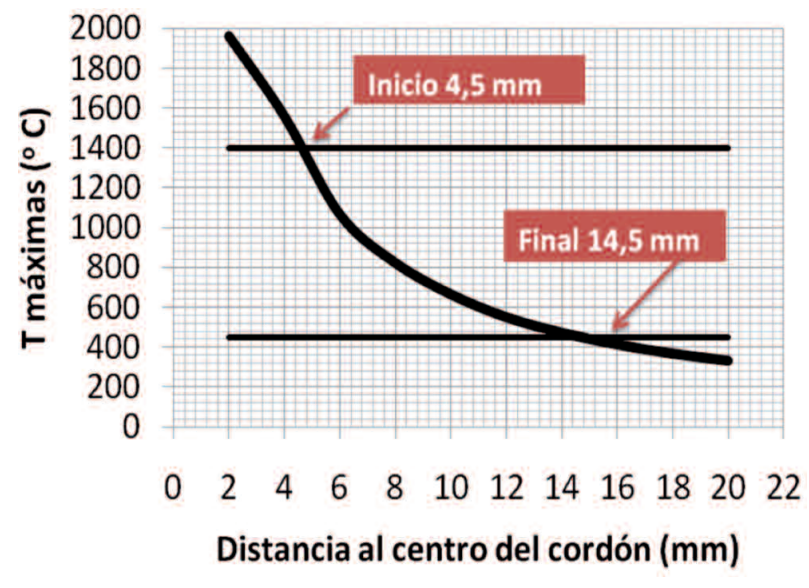

Figura 7. Dimensión de Z.A.T.

Figure 7. The HAZ dimension.

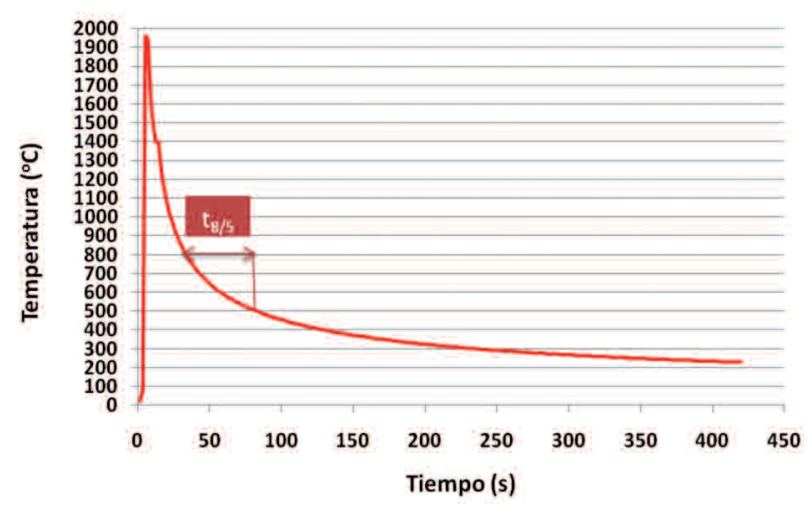

Figura 8. Cálculo del tiempo de enfriamiento.

Figure 8. Cooling time evaluation.

calentamiento. Lógicamente, el enfriamiento disminuye con la distancia al cordón de soldadura.

Uno de los parámetros más importantes relacionado con la afección térmica de los procesos de soldeo se define como el tiempo máximo en el que cualquier punto del metal base permanece a temperaturas comprendidas entre $800^{\circ} \mathrm{C}$ y $500^{\circ} \mathrm{C}, \mathrm{t}_{8 / 5}$. Dicho parámetro se ha calculado a partir de los datos arrojados por el método numérico. Para ello se ha considerado la línea de temperaturas que está más cercana al eje del cordón, en este caso a la distancia de $2 \mathrm{~mm}$, y se calcula la diferencia de tiempos entre los puntos de $800^{\circ} \mathrm{C}$ y $500^{\circ} \mathrm{C}$, tal como se indica en la figura 8 .

En la figura 9 se indican los resultados experimentales obtenidos en puntos situados a tres distancias diferentes del cordón de soldadura.

En la figura 10 se establece la comparación de resultados teóricos y experimentales para un punto situado a $5 \mathrm{~mm}$ del centro del cordón de soldadura. En

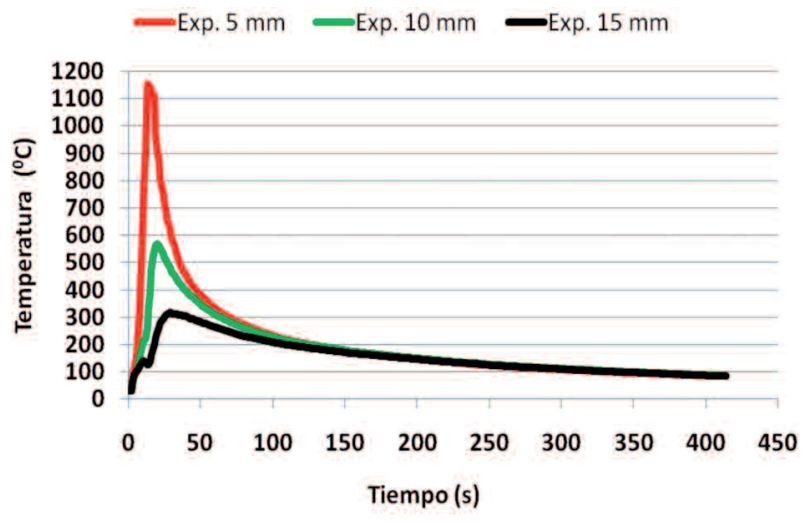

Figura 9. Resultados del procedimiento experimental.

Figure 9. Experimental results.

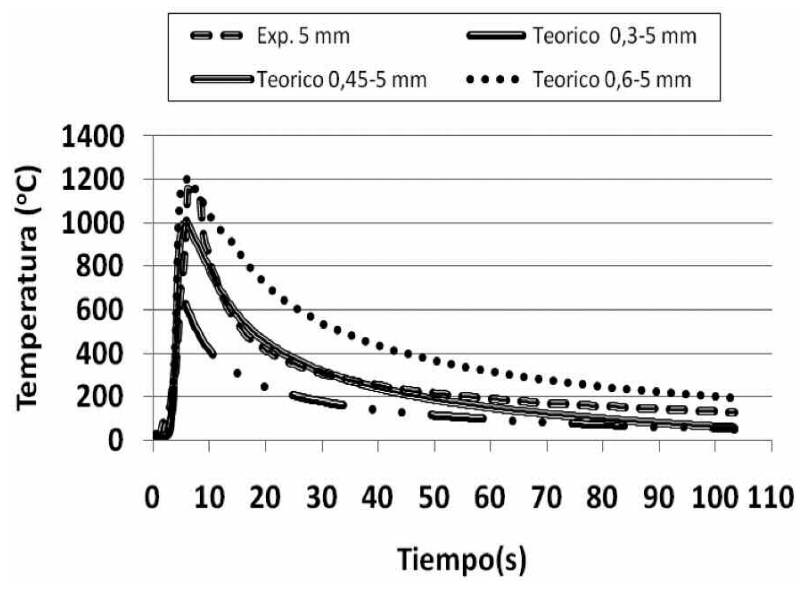

Figura 10. Grado de acuerdo entre resultados experimentales y numéricos.

Figure 10. Agreement between the experimental and numerical results.

la simulación numérica se han obtenido la temperatura del ciclo térmico de dicho punto considerando tres valores diferentes para el rendimiento térmico de la operación. Como puede observarse, el calentamiento y la temperatura máxima alcanzada se ajustan muy bien teóricamente si se considera un rendimiento de 0,6 para la operación. Este rendimiento está considerado como habitual para operaciones similares a la descrita ${ }^{[15]}$. Se puede observar que rendimientos menores conducen a obtener valores máximos de la temperatura inferiores, aunque no afecta significativamente al tiempo de calentamiento.

Si se comparan el ciclo térmico del modelo matemático y el obtenido experimentalmente, (Fig. 10) se puede apreciar que la velocidad de enfriamiento inicial es mayor en éste último. Se observa que la 
velocidad de enfriamiento de la experimental es más grande que la teórica, por tanto, el tiempo de enfriamiento es menor en la experimental que en la curva teórica, y esto se justifica ya que el flujo de calor en la simulación numérica es bidimensional, no se considera flujo de calor en la dirección del eje Z y además en la simulación se ha estudiado la ecuación de conducción de calor y se han despreciado las perdidas por radiación y convección. Además en el caso del proceso TIG el gas de protección inerte produce un efecto refrigerante sobre el baño de fusión constatado experimentalmente por algunos autores ${ }^{[17]}$.

La dificultad que supone acceder a los puntos muy cercanos al cordón de manera experimental ${ }^{[18]}$ hace especialmente útil el método numérico.

\section{CONCLUSIONES}

- El estudio de los ciclos térmicos en soldadura es un campo de investigación amplio y complejo dentro del cual se aplican diferentes herramientas, desde las soluciones analíticas hasta los modelos computacionales y su validación experimental. En este trabajo de investigación se ha implementado en VBA un procedimiento numérico basado en el método de diferencias finitas de Crank-Nicholson y adaptado al estudio de la difusión térmica en soldadura por fusión. En los cálculos, a diferencia de los métodos analíticos, se tiene en cuenta la licuación de la junta estimando la penetración, y se pueden utilizar distintos perfiles de aporte térmico, así como geometrías y trayectorias complejas. La disponibilidad y la extensión del programa soporte hace que no se requieran especiales conocimientos informáticos para su implementación y uso. Partiendo de los parámetros que definen el comportamiento térmico de un material, de las dimensiones de las chapas a soldar y de las variables del proceso, se puede obtener y representar gráficamente el campo completo de temperaturas durante la operación de soldeo.

- El método presentado se ha validado experimentalmente, aunque se ha detectado una ligera desviación de la curva de enfriamiento teórica respecto a la experimental debido a las hipótesis establecidas en el modelo. No se considera en éste las pérdidas de calor por convección y por radiación, lo que justifica las desviaciones encontradas. Una de las limitaciones del modelo matemático es que solamente se puede aplicar a chapas delgadas donde el flujo de calor es bidimensional, es decir, que la conducción del calor se efectúa en las direcciones paralelas al plano de la chapa.

\section{Agradecimientos}

Los autores del trabajo agradecen al Laboratorio de Ciencia e Ingeniería de Materiales del Instituto de Desarrollo Regional de Albacete de la Universidad de Castilla-La Mancha y a la Escuela de Arquitectura e Ingeniería de Edificación de la Universidad Politécnica de Cartagena el apoyo recibido en la realización del mismo.

\section{REFERENCIAS}

[1] D. Rosenthal, Transactions ASME, 68 (1946) 849-1.946.

[2] D. Rosenthal, Weld. J. 20 (1941) 220-234.

[3] J.C. Suárez, Tesis Doctoral, Facultad de Ciencias Químicas, Universidad Complutense de Madrid, 1990.

[4] J.C. Suárez, E. Carrillo, and F. Molleda, Rev. Metal. Madrid 21 (1991) 65-72.

[5] J. C. Suárez, F. Molleda, and J. M. Gomez de Salazar, Mater. Charact. 28 (1992) 3-13.

[6] N.T. Nguyen, A. Ohta, K. Matsuoka, N. Suzuki, and Y. Maeda, Weld. J. 78 (1999) 265-274.

[7] N.T. Nguyen, Y. W. Mai, S. Simpson, and A. Ohta, Weld. J. 83 (2004) 82-93.

[8] J. Goldak, A. Chakravarti, and M. Bibby, Metall. Trans. B-Process Metallurgy, 15 (1984) 299-305.

[9] J. Goldak, M. Bibby, J. Moore, R. House, and B. Patel, Metall. Trans. B-Process Metallurgy, 17 (1986) 587-600.

[10] J. A. Goldak, Computational Welding Mechanics, $1 \stackrel{\text { a }}{ }$ Ed. Springer, 2005.

[11] F.G. Lu, S. Yao, S.N. Lou, and Y.B. Li, Comp. Mater. Sci. 29 (2004) 371-378.

[12] T. Deb-Roy, J. Szekely, and T.W. Eagar, Mater. Sci. Eng. 56 (1982) 181-193.

[13] D. Alcaraz, F. Alhama, and J. A. Moreno, Rev. Metal. Madrid 41 (2005) 299-303.

[14] F. Alhama, D. Alcaraz, and C.F. GonzalezFernández, Rev. Metal. Madrid 41 (2005) 304 308.

[15] G. Di Caprio, Los aceros inoxidables, 2 ${ }^{\underline{a}}$ Ed. Grupinox, Milano, Italy, 1999.

[16] J. Crank and P. Nicolson, Proceedings of the Cambridge Philosophical Society 43 (1947) 50-67.

[17] V. Miguel, E.J. Martínez-Conesa, and M. Estrems, XII Congreso Internacional de Ingeniería de Proyectos, Vol. 1, Zaragoza, España, 2008, pp. 245-255.

[18] V. Miguel, A. Martinez, M. C. Manjabacas, J. Coello, and A. Calatayud, AIP Conference Proceedings, 1.181, EE.UU., 2009, pp. 170-179. 